\title{
Gastrointestinal stromal tumor with a PDGFRA mutation masquerading as gastric plexiform fibromyxoma: A comparative clinicopathological study of two cases
}

\author{
JUN ZHOU* , JINGJING XU*, GUOZHONG JIANG, YIHUI MA, JINGWEN QI, WENCAI LI and DANDAN ZHANG \\ Department of Pathology, The First Affiliated Hospital of Zhengzhou University, Zhengzhou, Henan 450052, P.R. China
}

Received July 21, 2015; Accepted October 26, 2016

DOI: $10.3892 / \mathrm{ol} .2016 .5486$

\begin{abstract}
Gastric plexiform fibromyxoma (PF) is a rare mesenchymal tumor with a histologically distinctive multinodular pattern, dissimilar to conventional gastrointestinal stromal tumor (GIST). The current study presents one case of gastric PF, and one case of GIST with a platelet-derived growth factor receptor $\alpha$ (PDGFRA) mutation mimicking PF, and discusses their differential diagnoses. The two patients were a 51-year-old male with PF and a 47-year-old female with GIST, each of whom presented with an occupying lesion in the gastric antrum. Histologically, the two cases shared a rare and approximately unanimous morphological pattern of a prominent multinodular and plexiform figuration in the gastric wall, including mucoid matrix, short spindle cells and small caliber vascular elements, and areas of stromal tumor cells exhibited an epithelioid appearance. Immunohistochemistry revealed that the PF tumor cells were positive for smooth muscle actin (SMA), but negative for mast/stem cell growth factor receptor (KIT), GIST-1 (DOG1), cluster of differentiation (CD) 34, S-100, desmin and cytokeratin AE1/AE3. The case of GIST expressed KIT and DOG1, but was negative for SMA, CD34, S-100, desmin and AE1/AE3. In addition, the GIST case, which was observed to harbor a D842V mutation in exon 18 of PDGFRA, was demonstrated to be genetically distinct from $\mathrm{PF}$. The cases presented in the current study were uncommon in that GIST exhibited a plexiform appearance that mimicked the histology of the rare PF tumor; therefore, GIST must be considered and discounted first when determining a differential diagnosis for a gastrointestinal mesenchymal neoplasm.
\end{abstract}

Correspondence to: Dr Dandan Zhang, Department of Pathology, The First Affiliated Hospital of Zhengzhou University, 1 Jianshe East Road, Zhengzhou, Henan 450052, P.R. China

E-mail: zhoujunlongch@126.com

*Contributed equally

Key words: plexiform fibromyxoma, gastrointestinal stromal tumor, platelet-derived growth factor receptor $\alpha$, differential diagnosis, sequencing

\section{Introduction}

Gastric plexiform fibromyxoma (PF), also known as plexiform angiomyxoid myofibroblastic tumor(PAMT), is a mesenchymal tumor that typically develops in the pyloric antrum or duodenal bulb with a multinodular pattern infiltrating the muscularis propria $(1,2)$. The nodules are composed of bland spindle cells embedded in a myxoid matrix with myofibroblastic differentiation and small thin blood vessels (2). Epidemiologically, PF usually affects young or middle-aged males and females $(1,2)$. $\mathrm{PF}$ is frequently misdiagnosed as gastrointestinal stromal tumor (GIST) in routine clinical practice, but the prognosis is good with little recurrence reported (1-8). GIST is a more prevalent mesenchymal tumor involving the gastrointestinal tract, including the stomach $(60 \%)$ and small intestinal tract $(30 \%)$, and other common locations include the pancreas, gallbladder and appendix (9). Activation of KIT (80\%) and platelet-derived growth factor receptor $\alpha(P D G F R A, 7 \%)$ are the typical molecular characteristics of this tumor (10). The biological behaviors of GIST are varied and features including the anatomic location, tumor size and mitotic activity are able to aid the evaluation of patient prognosis (11). The morphology of GIST frequently exhibits a sheet pattern with spindle or epithelioid cells, but plexiform architecture and the presence of myxoid matrix are uncommon (12).

The current study presents a case of PF and a case of GIST masquerading as PF by exhibiting a distinctive multinodular figuration due to a PDGFRA mutation. Therefore, the present study demonstrates the necessity for considering and eliminating the possibility of GIST first, when determining the differential diagnosis of gastrointestinal mesenchymal tumors.

\section{Case report}

Case 1. A 51-year-old male was admitted to the First Affiliated Hospital of Zhengzhou University (Zhengzhou, China) in January 2013 due to epigastric discomfort and an intermittent burning sensation in the chest, without nausea, acid reflux or stomach-ache. A gastroscopy and a computed tomography (CT) scan revealed a mass located in the atrium of the greater curvature of the stomach and protruding into the gastric cavity, creating a narrowed outlet (Fig. 1). No significant biomarkers were identified, including $\alpha$-fetoprotein, carcinoembryonic antigen and carbohydrate antigen 19-9, and there was no 
relevant family medical history. Total resection of the tumor was performed, and the patient had no evidence of relapse or metastasis for 15 months following surgery.

The dimensions of the submucosal tumor were $4 \times 3 \times 1.8 \mathrm{~cm}$; the cut surface appeared pale tan and gelatinous, and subtle intramural nodules were visible. The surgical specimen was routinely fixed in $4 \%$ buffered formalin, embedded in paraffin and then hematoxylin and eosin (H\&E) staining was performed using the Leica ST 5010 Autostainer XL (Leica Microsystems, Inc., Buffalo Grove, IL, USA). Immunohistochemical studies were performed with commercial antibodies using the Ventana BenchMark XT instrument (Ventana Medical Systems, Inc., Tucson, AZ, USA) according to the manufacturer's protocol. Briefly, the tissue sections were deparaffinized using EZ prep solution (Ventana Medical Systems, Inc.), followed by heat-induced epitope retrieval using $\mathrm{CC} 1$ solution $(20 \mathrm{~min}$ at $95^{\circ} \mathrm{C}$; Ventana Medical Systems, Inc.). Subsequently, the slides were incubated with primary antibodies for $1 \mathrm{~h}$ at $37^{\circ} \mathrm{C}$ and Ventana anti-rabbit secondary antibody (ultraView universal HRP multimer, prediluted; included in the ultraView Universal DAB Detection kit, no. 760-500; Ventana Medical Systems, Inc.) for $8 \mathrm{~min}$ at $37^{\circ} \mathrm{C}$. The immunoreaction was detected under a light microscope (BX43; Olympus Corporation, Tokyo, Japan) following use of the ultraView Universal DAB Detection kit and counterstaining with hematoxylin and bluing reagent (Ventana Medical Systems, Inc.). The primary antibodies included KIT (no. A4502; polyclonal; dilution, 1:200; Dako, Glostrup, Denmark), Dog-1 (no. MONX11114; clone K9; dilution, 1:200; Novocastra; Leica Microsystems Inc.), S-100 (no. Z0311; polyclonal; dilution, 1:400; Dako), SMA (no. M0851; clone 1A4; dilution, 1:100; Dako) desmin (no. M0760; clone D33; dilution, 1:50; Dako), Ki-67 (no. M7248; clone MIB; dilution 1:100; Dako), cluster of differentiation (CD) 34 (no. 550760; clone MY10, dilution, 1:100; BD Biosciences, San Jose, CA, USA) and cytokeratin AE1/AE3 (no. M3515; clone AE1+AE3; dilution 1:100; Dako). Sanger sequencing was performed to detect the status of exons 9,11, 13 and 17 of KIT and exons 12 and 18 of PDGFRA. The coding regions of these exons were amplified by polymerase chain reaction (PCR) using HotStart Taq DNA polymerase. The reaction conditions and primers were used according to previously published protocol (13-15). The PCR products were directly sequenced using BigDye Terminator v3.1 Cycle Sequencing kit (Applied Biosystems; Thermo Fisher Scientific, Inc.) according the manufacturer's protocol on the ABI 4500Dx, and analyzed using ABI Prism 3500Dx DNA Sequence Analysis Software version 4.0. The products were sequenced with forward and reverse primers, as previously reported $(16,17)$. Histological analysis revealed that the tumor had a plexiform and multinodular involvement in the muscularis propria (Fig. 2A). The nodules were variable in size with a fine demarcation or infiltrative margin from the remaining normal tissues, a number of which had coalesced to form sheets. Within the nodules, the bland spindle cells (with tapering ends, round or oval nuclei, fine chromatin, indistinct nucleoli, an eosinophilic or amphophilic cytoplasm, and discrete cell borders) were dispersed in the matrix (Fig. 2B). Necrosis and mitotic bodies were not detectable using a light microscope (magnification, 200x; BX43; Olympus Corporation). An arborizing vascular network of small capillaries was

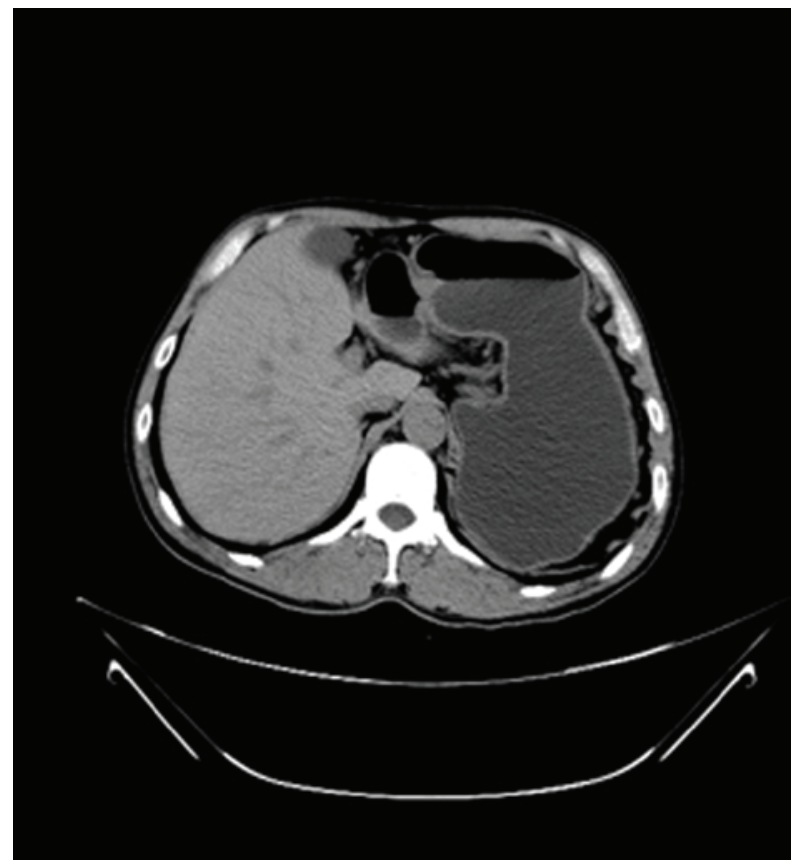

Figure 1. Case 1: Computed tomography revealed a mass located in the atrium of the greater curvature protruding into the gastric cavity.

observed in the tissue (Fig. 2B). In addition, some tumor cells with indistinct borders were embedded in the dense collagenous matrix deficient in mucus, and exhibited an epithelioid appearance reminiscent of epithelioid GIST (Fig. 2C). The tumor cells demonstrated immunoreactivity for smooth muscle actin (SMA; Fig. 2D), but were negative for mast/stem cell growth factor receptor (KIT), GIST-1 (DOG1), CD34, S-100, desmin and cytokeratin AE1/AE3; staining for CD34 delineated the capillary network. The Ki- 67 proliferation index was $\sim 1 \%$, and KIT (exon 9, 11, 13 and 17) and PDGFRA (exon 12 and 18) genetic mutations were not identified. The final diagnosis was determined to be PF.

Case 2. A 47-year-old female was admitted to The First Affiliated Hospital of Zhengzhou University in April 2013 due to a 2-month history of intermittent abdominal distension, acid reflux and heartburn. A CT scan revealed a mass $(48 \times 36 \mathrm{~mm}$ in the maximum cross-section) located in the gastric antrum of the lesser curvature (Fig. 3). No significant biomarkers or relevant family medical history were identified. The neoplasm was completely resected by a distal gastrectomy without adjuvant therapies, and the patient had a favorable prognosis without relapse or metastasis for 12 months.

The submucosal tumor measured $4 \times 3 \times 3 \mathrm{~cm}$, and the cut surface appeared gray and subtly mucoid. The resected specimen was examined using H\&E and immunohistochemical staining, and the molecular testing of KIT and PDGFR was performed, according to the aforementioned protocol in case 1 . Under a light microscope (BX43; Olympus Corporation), the tumor exhibited a nodular growth pattern involving the muscularis propria (Fig. 4A). The nodules varied in size and merged together in some areas; they were principally composed of numerous randomly arranged spindle cells, short capillaries and dilated small veins in a myxoid matrix (Fig. 4B). Significant hemorrhage was observed in some of the nodules, but evidence 

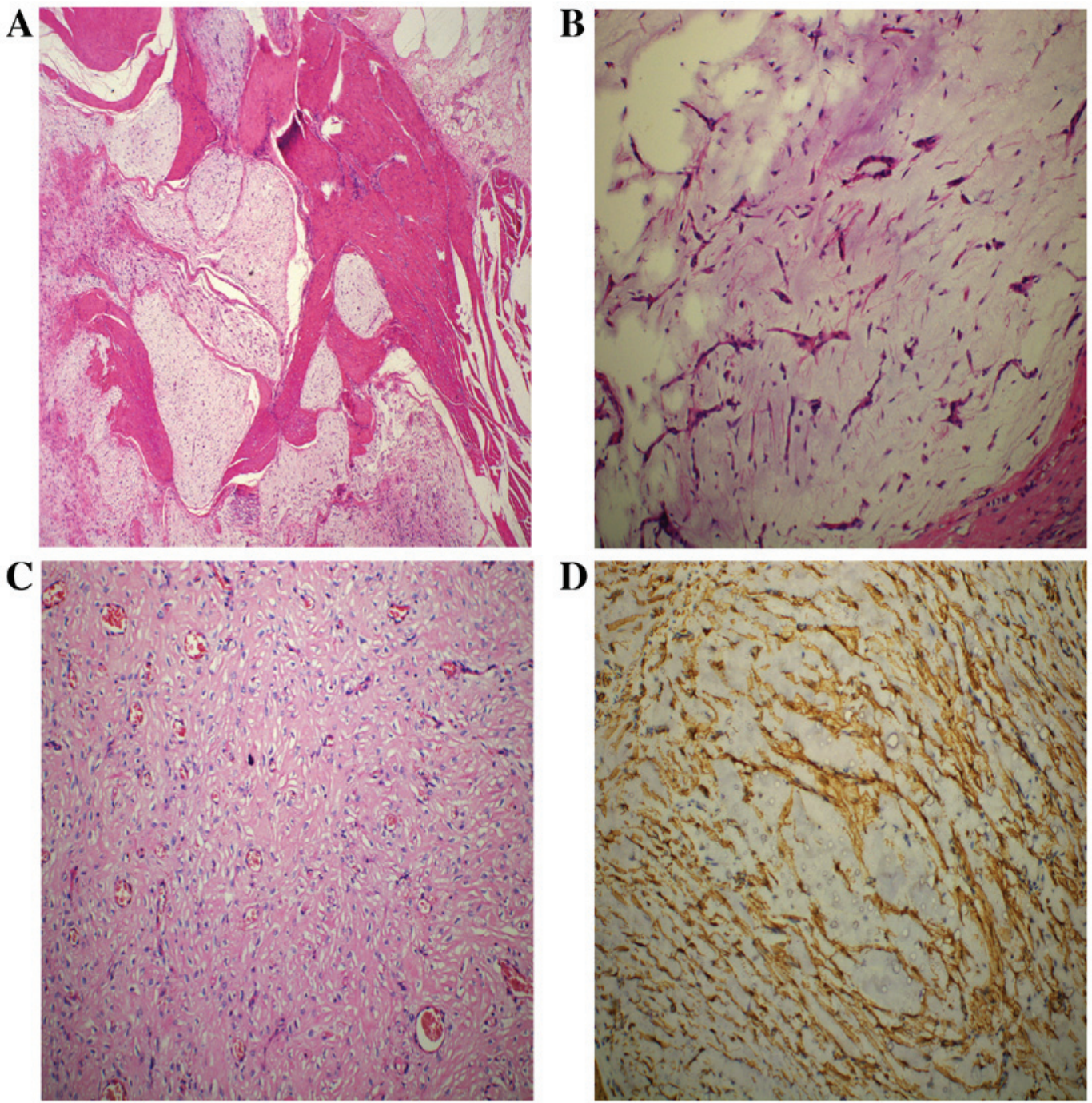

Figure 2. (A) Histological analysis of PF tissues revealed multinodular involvement through the muscularis propria with specific areas of coalescence (staining, $\mathrm{H} \& \mathrm{E}$; magnification, 40x). (B) Within the nodules of the PF, bland spindle cells were distributed in a myxoid matrix with a prominent arborizing vascular network of small capillaries (staining H\&E; magnification, 200x). (C) Some PF tumor cells were embedded in a dense collagenous matrix (staining H\&E; magnification, 200x). (D) The PF tumor cells were positive for smooth muscle actin expression (staining H\&E; magnification, 200x). PF, plexiform fibromyxoma; H\&E, hematoxylin and eosin.

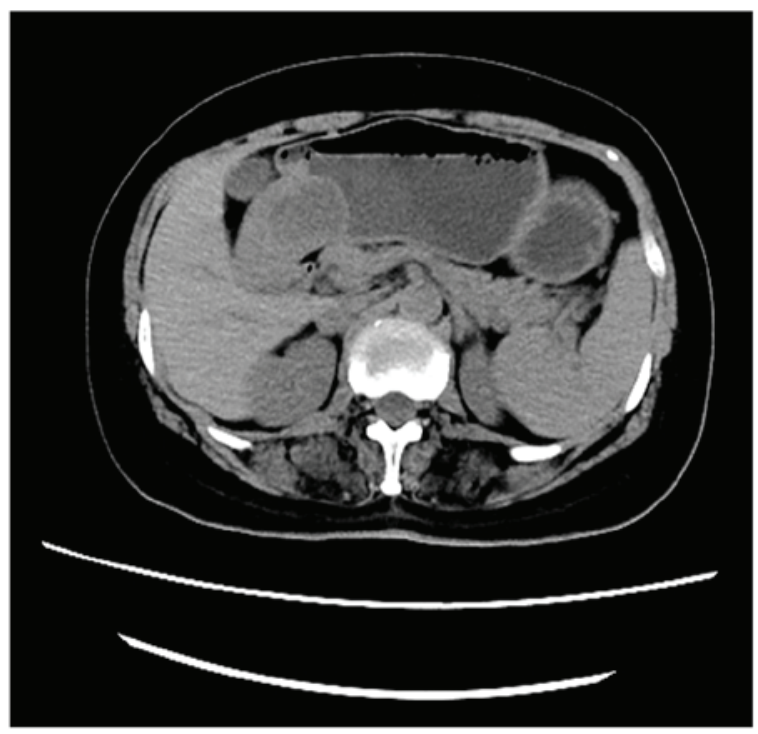

Figure 3. Case 2: Computed tomography scan revealed a mass located in the antrum of the lesser curvature of the stomach. of necrosis and mitotic bodies was absent. In addition, the tumor cells in some nodules exhibited prominent cytoplasmic vacuoles, similar to the vacuoles typically observed in epithelioid GIST (Fig. 4C). All these features are similar to those observed in $\mathrm{PF}$, as depicted in case 1 . However, the diagnosis of GIST must be eliminated for soft tissue neoplasms in the gastrointestinal tract, due to the changeable morphological patterns observed in this disease. Accordingly, immunohistochemistry was performed, demonstrating that the tumor cells were positive for KIT (Fig. 5A) and DOG1 (Fig. 5B), but were negative for SMA, CD34, S-100, desmin and AE1/AE3. The Ki-67 proliferation index was $\sim 3 \%$. Sequencing revealed a D842V mutation in exon 18 of the PDGFRA gene; however, mutations in exon 12 of the PDGFRA and KIT genes were not detected (Fig. 6). Therefore, the final diagnosis was determined to be GIST.

\section{Discussion}

$\mathrm{PF}$ is a rare and benign cancer that exclusively develops in the stomach, particularly in the gastric antrum, and has previously 
A

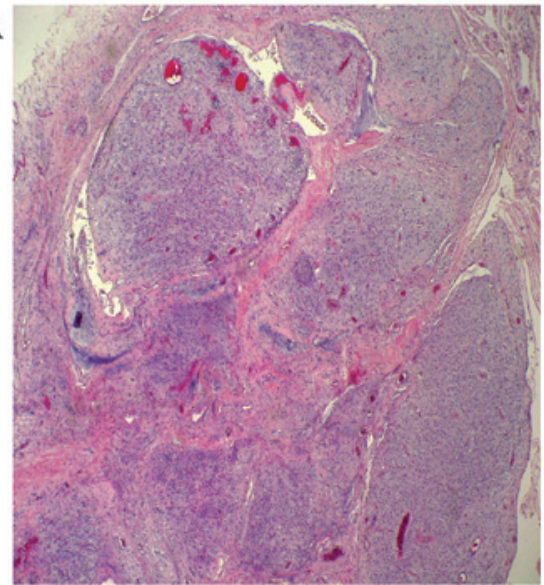

B

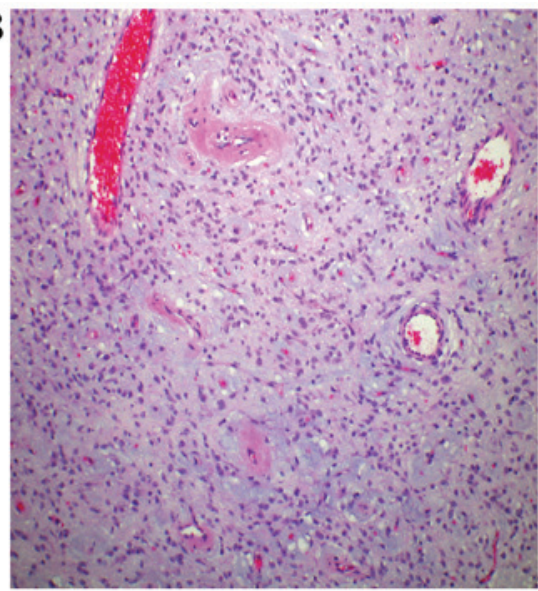

C

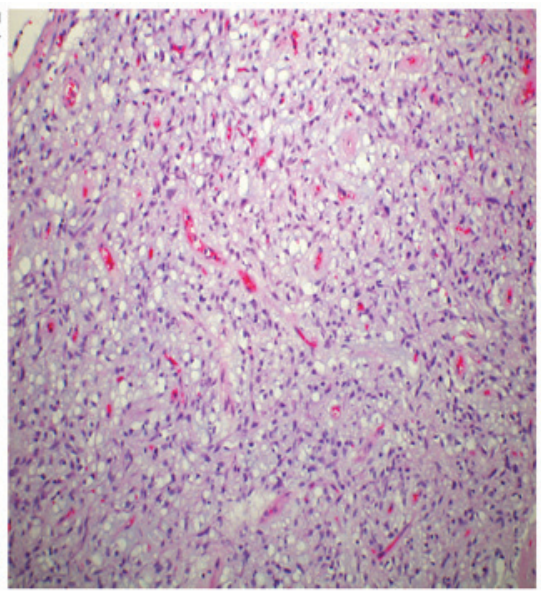

Figure 4. (A) GIST presented with a nodular growth pattern involving the muscularis propria (staining, H\&E; magnification, 40x). (B) The nodules of GIST were composed of numerous randomly arranged spindle cells and proliferative capillaries and small veins in the myxoid matrix (staining, H\&E; magnification, 200x). (C) The GIST cells in certain nodules with prominent cytoplasmic vacuoles exhibited epithelioid features (staining, H\&E; magnification, 200x). GIST, gastrointestinal stromal tumor; H\&E, hematoxylin and eosin.

A

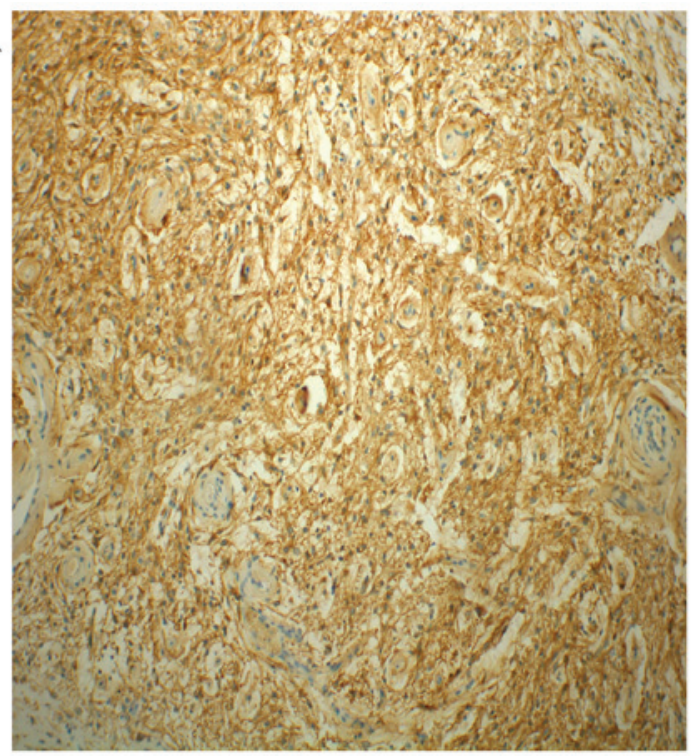

B

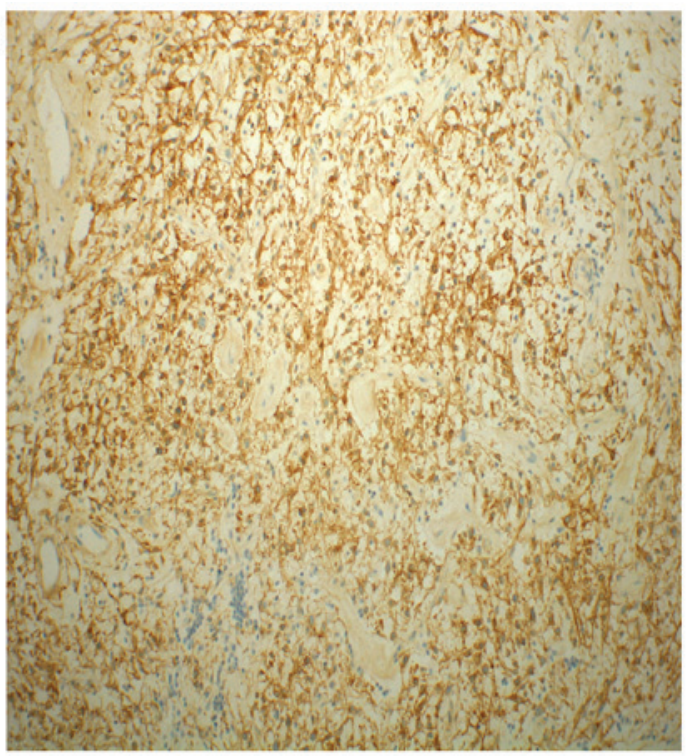

Figure 5. Immunohistochemistry determined that the gastrointestinal stromal tumor cells were positive for (A) KIT and (B) DOG1 expression (magnification, 200x).

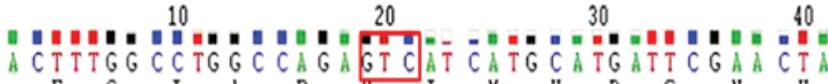

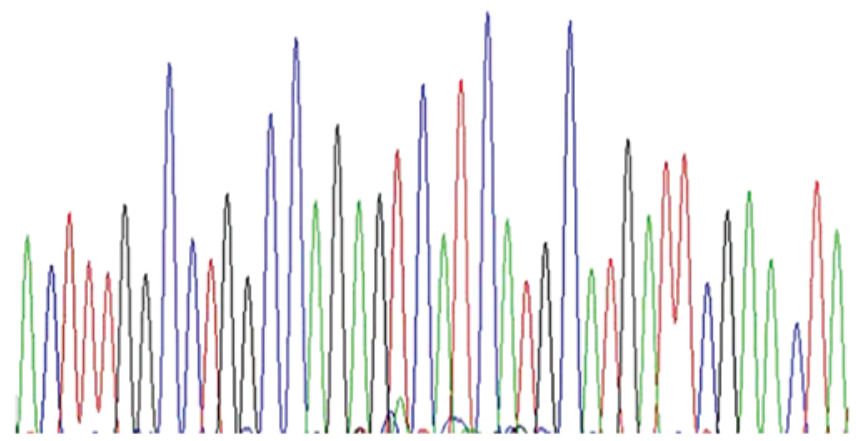

Figure 6. In the gastrointestinal stromal tumor cells, a D842V mutation was identified in exon 18 of the platelet-derived growth factor receptor $\alpha$ gene using Sanger sequencing. A 'GAC' codon was substituted for a 'GTC' (highlighted by the red frame). been designated as myxoma, giant myxoma, fibromyxoma and plexiform angiomyxoid myofibroblastic tumor $(1,3,18,19)$. $\mathrm{PF}$ has no significant gender distribution and primarily presents in young and middle-aged adults (7-75 years, mean 41 years) $(2,20)$. Patients typically present with gastrointestinal bleeding, anemia, ulcer, hematemesis, an occasional gastric outlet obstruction or other nonspecific upper digestive tract symptoms $(5,8)$. The tumors usually range in size from 2 to $15 \mathrm{~cm}$ (median, $5.5 \mathrm{~cm}$ ), and have a multinodular, pale appearance and a mucoid cut surface (2).

PF exhibits a histologically distinctive multinodular plexiform architecture involving the muscularis propria and the serosa with an infiltrative margin $(2,21)$. The nodules are usually composed of an abundant myxoid matrix, prominent arborizing capillaries, and scattered spindle cells with small nuclei, inconspicuous nucleoli and an eosinophilic cytoplasm $(1,2)$. Mitotic activity and necrosis are scarcely 
apparent $(1,22)$. Immunohistochemistry typically demonstrates that the PF tumor cells are consistently positive for SMA, but negative for KIT, DOG1, CD34, S-100, desmin and caldesmon, indicating myofibroblastic differentiation and a low Ki-67 proliferation index (20). Previous studies and follow-up data indicate that PF typically has a favorable prognosis and that a conservative treatment involving tumor resection is sufficient (1-8).

As the most common mesenchymal neoplasm in the gastrointestinal tract, GISTs are considered to originate from the interstitial cells of Cajal (1). They are primarily driven by the mutations in KIT or PDGFRA, which account for $80 \%$ and $5-8 \%$ of cases, respectively (23). GISTs may develop at any age, but typically present in middle-aged or elderly patients, with a median age of onset of $\sim 60$ years without specific gender distribution (24).

By contrast to PFs, GISTs may involve any tissues along the gastrointestinal tract, including the gastric antrum, and have varied biological behaviors that may be assessed using a specialized system of risk stratification based on anatomic site, tumor size and mitotic activity $(11,25)$. A variable histological morphology of GIST tissues may be observed, including completely spindle shaped, entirely epithelioid shaped or pleomorphic cell cytomorphologies (25). The spindle cell GISTs of the stomach are often arranged in fascicles with round or elongated nuclei, fine chromatin, inconspicuous nucleoli, a moderate to abundant quantity of eosinophilic cytoplasm, relatively infrequent mitotic bodies and no cytological pleomorphisms $(25,26)$. Occasionally, gastric GISTs may exhibit prominent paranuclear vacuoles and nuclear palisading that mimics schwannoma (26). Epithelioid GIST variants exhibit a sheet-like or nested growth pattern with round or polygonal cells, vesicular chromatin and an eosinophilic, clear or vacuolated cytoplasm, which may occasionally result in a conspicuous signet ring form (27). Extremely rare subsets of gastric GISTs have a plexiform growth pattern, particularly in the case of 'pediatric-type' GIST or patients with Carney triad or Carney-Stratakis syndrome. These cases exhibit distinctive features including female predilection, young age, epithelioid tumor cells or a multifocal growth pattern, frequent lymph node metastasis and serial tumor occurrence, but relatively indolent behavior even with metastasis, with a low number of cases being fatal $(2,28,29)$. The presence of these characteristics indicates that a diagnosis of GIST may be considered. Other lesions, including extra-adreanal paragonglioma, pulmonary chondroma and the mutation of succinate dehyrogenase (SDH) complex B, SDH complex C, or SDH complex D (the genetic basis of Carney-Stratakis syndrome) may also be detected (30). Similar to the aforementioned literature $(1,28,29)$, the patient discussed in case 2 was diagnosed with GIST and had a favorable prognosis; however, no evidence of a Carney complex or of other hereditary diseases was identified.

GIST tissues frequently exhibit variably sized nodules with irregular margins or broad bands involving the muscularis propria; the nodules are often composed of variable areas of epithelioid or mixed spindle and epithelioid cell morphology, with or without myxoid or collagen matrix (29).

The majority of GISTs have constitutively activating mutations in the KIT gene with four mutation hotspots, including on exons $9,11,13$ and 17, accounting for $65,10,1$ and $1 \%$ of GISTs, respectively (31). The small molecular inhibitor imatinib has been used as the first-line drug for the treatment of GIST with KIT mutations (32). However, a minority of GISTs $(\sim 5 \%)$ have a PDGFRA mutation, with three mutation hotspots, of which exon 18 is the most frequently affected (almost always the $\mathrm{D} 842 \mathrm{~V}$ mutation), contributing to the clinical resistance to imatinib and a more favorable disease course $(23,31,33)$. Similarly, the case of GIST presented in the current study had a good prognosis following tumor resection without targeted therapy. GISTs with an epithelioid phenotype or a plexiform growth pattern usually exhibit a PDGFRA mutation, and are preferentially located in the stomach (33). Immunohistochemistry has demonstrated that $\sim 80 \%$ of GISTs express KIT (CD117) and CD34, but SMA S-100 and desmin are expressed in 25,5 and $<1 \%$ of GISTs, respectively (34).

The case of gastric GIST discussed in the present study exhibited a prominent multinodular or plexiform pattern with myxoid stroma and proliferated capillaries, reminiscent of PF. However, the case of PF exhibited a more abundant mucous matrix and a distinctively elongated vascular network that was not present in the GIST tissues. The nodules of the GIST consisted of sheets of round cells with prominent cytoplasmic vacuoles, suggesting a possible diagnosis of a GIST with a distinctive growth pattern, rather than PF; however, in PF tissues, the presence of some tumor cells embedded in the dense collagenous stroma with epithelioid features may superficially resemble GIST. Immunostaining for KIT and DOG1 and the detection of a D842V mutation in exon 18 of the PDGFRA gene distinguishes these two types of gastric cancer.

In conclusion, $\mathrm{PF}$ is a rare benign mesenchymal neoplasm that frequently develops in the gastric antrum, and exhibits a prominent plexiform growth pattern with an abundant myxoid matrix and a distinctive vascular network. However, a small subset of gastric GISTs that present with myxoid and multinodular histological features may masquerade as PF. Therefore, GIST must be considered and rejected first when determining a differential diagnosis for gastrointestinal mesenchymal neoplasms.

\section{Acknowledgements}

This study was supported by The First Affiliated Hospital of Zhengzhou University.

\section{References}

1. Takahashi Y, Shimizu S, Ishida T, Aita K, Toida S, Fukusato T and Mori S: Plexiform angiomyxoid myofibroblastic tumor of the stomach. Am J Surg Pathol 31: 724-728, 2007.

2. Miettinen M, Makhlouf HR, Sobin LH and Lasota J: Plexiform fibromyxoma: A distinctive benign gastric antral neoplasm not to be confused with a myxoid GIST. Am J Surg Pathol 33: 1624-1632, 2009

3. Fukunaga M: Gastric fibromyxoma, a distinct entity of pure fibroblastic tumor-an ultrastructural study. APMIS 112: 304-308, 2004.

4. Ikemura M, Maeda E, Hatao F, Aikou S, Seto Y and Fukayama M: Plexiform angiomyxoid myofibroblastic tumor (PAMT) of the stomach. A case report focusing on its characteristic growth pattern. Int J Clin Exp Pathol 7: 685-689, 2014.

5. Lee PW, Yau DT, Lau PP and Chan JK: Plexiform fibromyxoma (plexiform angiomyxoid myofibroblastic tumor) of stomach: An unusual presentation as a fistulating abscess. Int J Surg Pathol 22: 286-290, 2014. 
6. Kim A, Bae YK, Shin HC and Choi JH: Plexiform angiomyxoid myofibroblastic tumor of the stomach: A case report. J Korean Med Sci 26: 1508-1511, 2011

7. Kang Y, Jung W, Do IG, Lee EJ, Lee MH, Kim KM and Choi J: Plexiform angiomyxoid myofibroblastic tumor of the stomach: Report of two cases and review of the literature. Korean J Pathol 46: 292-296, 2012.

8. Takahashi Y, Suzuki M and Fukusato T: Plexiform angiomyxoid myofibroblastic tumor of the stomach. World J Gastroenterol 16 : 2835-2840, 2010.

9. de la Roza G, Naqvi A and Clark K: Gastrointestinal stromal tumors presenting as a prostatic mass. Can J Urol 16: 4502-4506, 2009.

10. Hirota S, Isozaki K, Moriyama Y, Hashimoto K, Nishida T, Ishiguro S, Kawano K, Hanada M, Kurata A, Takeda M, et al Gain-of-function mutations of c-kit in human gastrointestinal stromal tumors. Science 279: 577-580, 1998.

11. Miettinen M and Lasota J: Gastrointestinal stromal tumors: Pathology and prognosis at different sites. Semin Diagn Pathol 23: 70-83, 2006.

12. Miettinen $\mathrm{M}$ and Lasota J: Gastrointestinal stromal tumors: Review on morphology, molecular pathology, prognosis, and differential diagnosis. Arch Pathol Lab Med 130: 1466-1478, 2006.

13. Tryggvason G, Hilmarsdottir B, Gunnarsson GH, Jónsson JJ, Jónasson JG and Magnusson MK: Tyrosine kinase mutations in gastrointestinal stromal tumors in a nation-wide study in Iceland. APMIS 118: 648-656, 2010.

14. Went PT, Dirnhofer S, Bundi M, Mirlacher M, Schraml P, Mangialaio S, Dimitrijevic S, Kononen J, Lugli A, Simon R and Sauter G: Prevalence of KIT expression in human tumors. J Clin Oncol 22: 4514-4522, 2004.

15. Buleje J, Acosta Ó, Guevara-Fujita M, Enriquez Y, Taxa L, Machicado E, Lizaraso-Caparó F and Fujita R: Mutational profile of KIT and PDGFRA genes in gastrointestinal stromal tumors in Peruvian samples. Rev Esp Enferm Dig 107: 72-78, 2015.

16. Antonescu CR, Sommer G, Sarran L, Tschernyavsky SJ, Riedel E, Woodruff JM, Robson M, Maki R, Brennan MF, Ladanyi M, et al: Association of KIT exon 9 mutations with nongastric primary site and aggressive behavior: KIT mutation analysis and clinical correlates of 120 gastrointestinal stromal tumors. Clin Cancer Res 9: 3329-3337, 2003.

17. Heinrich MC, Corless CL, Duensing A, McGreevey L, Chen CJ, Joseph N, Singer S, Griffith DJ, Haley A, Town A, et al PDGFRA activating mutations in gastrointestinal stromal tumors. Science 299: 708-710, 2003.

18. Traverso JP and Vidal HJ: Stomach myxoma. Prensa Med Argent 43: 881-883, 1956 (In Spanish).
19. Faraoni H, Recagno Urruti ER and Escalante DA: Giant myxoma of the stomach. Sem Med 106: 135-136, 1955 (In Spanish).

20. Duckworth LV, Gonzalez RS, Martelli M, Liu C, Coffin CM and Reith JD: Plexiform fibromyxoma: Report of two pediatric cases and review of the literature. Pediatr Dev Pathol 17: 21-27, 2014.

21. Galant C, Rousseau E, Ho Minh Duc DK and Pauwels P: Re: Plexiform angiomyxoid myofibroblastic tumor of the stomach. Am J Surg Pathol 32: 1910; author reply 1912-1913, 2008.

22. Sing Y, Subrayan S, Mqadi B, Ramdial PK, Reddy J, Moodley MS and Bux S: Gastric plexiform angiomyxoid myofibroblastic tumor. Pathol Int 60: 621-625, 2010.

23. Eisenberg BL and Pipas JM: Gastrointestinal stromal tumor-background, pathology, treatment. Hematol Oncol Clin North Am 26: 1239-1259, 2012.

24. Quek R and George S: Gastrointestinal stromal tumor: A clinical overview. Hematol Oncol Clin North Am 23: 69-78.viii, 2009.

25. Kitamura Y: Gastrointestinal stromal tumors: Past, present, and future. J Gastroenterol 43: 499-508, 2008.

26. Miettinen $\mathrm{M}$ and Lasota J: Histopathology of gastrointestinal stromal tumor. J Surg Oncol 104: 865-873, 2011.

27. Suster S and Fletcher CD: Gastrointestinal stromal tumors with prominent signet-ring cell features. Mod Pathol 9: 609-613, 1996.

28. Zhang L, Smyrk TC, Young WF Jr, Stratakis CA and Carney JA: Gastric stromal tumors in Carney triad are different clinically, pathologically, and behaviorally from sporadic gastric gastrointestinal stromal tumors: Findings in 104 cases. Am J Surg Pathol 34: 53-64, 2010.

29. Rege TA, Wagner AJ, Corless CL, Heinrich MC and Hornick JL: 'Pediatric-type' gastrointestinal stromal tumors in adults: Distinctive histology predicts genotype and clinical behavior. Am J Surg Pathol 35: 495-504, 2011.

30. Pasini B, McWhinney SR, Bei T, Matyakhina L, Stergiopoulos S, Muchow M, Boikos SA, Ferrando B, Pacak K, Assie G, et al: Clinical and molecular genetics of patients with the Carney-Stratakis syndrome and germline mutations of the genes coding for the succinate dehydrogenase subunits SDHB, SDHC, and SDHD. Eur J Hum Genet 16: 79-88, 2008.

31. Joensuu H: Gastrointestinal stromal tumor (GIST). Ann Oncol 17 (Suppl 10): x280-x286, 2006

32. Siehl J and Thiel E: C-kit, GIST, and imatinib. Recent Results Cancer Res 176: 145-151, 2007.

33. Yang J, Du X, Lazar AJ, Pollock R, Hunt K, Chen K, Hao X, Trent J and Zhang W: Genetic aberrations of gastrointestinal stromal tumors. Cancer 113: 1532-1543, 2008.

34. Miettinen M, Sobin LH and Lasota J: Gastrointestinal stromal tumors of the stomach: A clinicopathologic, immunohistochemical, and molecular genetic study of 1765 cases with long-term follow-up. Am J Surg Pathol 29: 52-68, 2005. 